Supporting Information for:

\title{
Quantifying the dimensions of nanoscale organic surface layers in natural waters
}

\author{
C.T. Gibson ${ }^{1+}$, Ian J. Turner ${ }^{2}$, Clive J. Roberts ${ }^{3}$ and J.R.Lead ${ }^{1} *$ \\ * Corresponding author: j.r.lead@bham.ac.uk
}

${ }^{1}$ School of Geography, Earth and Environmental Sciences, University of Birmingham, Birmingham, B15 2TT, U.K.

${ }^{2}$ Biology Sciences Research Group, School of Education, Health and Science, University of Derby, Derby, DE22 1GB, UK.

${ }^{3}$ Laboratory of Biophysics and Surface Analysis, School of Pharmacy, The University of Nottingham, University Park NG7 2RD, UK

+ Current address: Laboratory of Biophysics and Surface Analysis, School of Pharmacy, The University of Nottingham, University Park NG7 2RD, UK

Supporting information for the article published in Environmental Science and Technology, prepared $7^{\text {th }}$ November 2006. There are four pages in total, including current page. Contains 2 figures. 


\section{Methodology}

Understanding reproducibility in the system.

The drift on the AFM retract and approach system was small enough (typically less than $500 \mathrm{~nm}$ ) so that, as long as we imaged a larger area than the "scratched" region, it could be easily located again. Figures S1 and S2 demonstrate how small the probe's positional drift is when the tip is retracted from the surface and then re-approached. The sample in these figures was $\mathrm{pH} 4.8$ with an exposure time to the humic solution of 2.5 hours. Figure S1 shows a tapping mode image with a 500 x $500 \mathrm{~nm}^{2}$ area that has been "scratched" using contact mode (highlighted inside the white circle on figure 2). The AFM probe was then retracted and the instrument switched to contact mode and then re-approached. A second 500 x $500 \mathrm{~nm}^{2}$ region was then "scratched" in contact mode. The tip was then retracted again and the instrument switched back to tapping mode and then re-approached but this time with a scan area of $3 \times 3 \mu \mathrm{m}^{2}$. Figure 3 shows the new tapping mode image with the second scratched area highlighted inside a black circle. The positions of SRHA particles near the scratched areas in figure S2 correspond to SRHA particles in figure S1 demonstrating that these images are of the same region. From figure 3 it can be seen that the probe movement when the tip is retracted and then re-approached is less than 500nm. This makes it a simple task to find the "scratched" regions when moving from tapping mode to contact and then back to tapping mode again. 


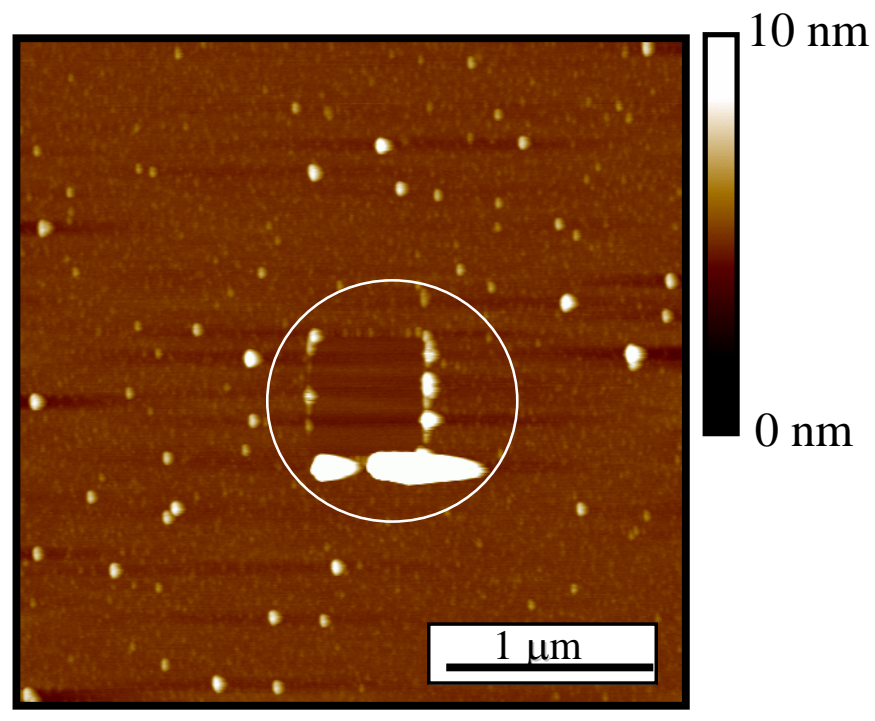

Figure S1. A tapping mode image of a humic layer that has had a 500 x $500 \mathrm{~nm}^{2}$ area scratched away in contact mode. The scratched area can be easily observed but has been highlighted inside a white circle. 


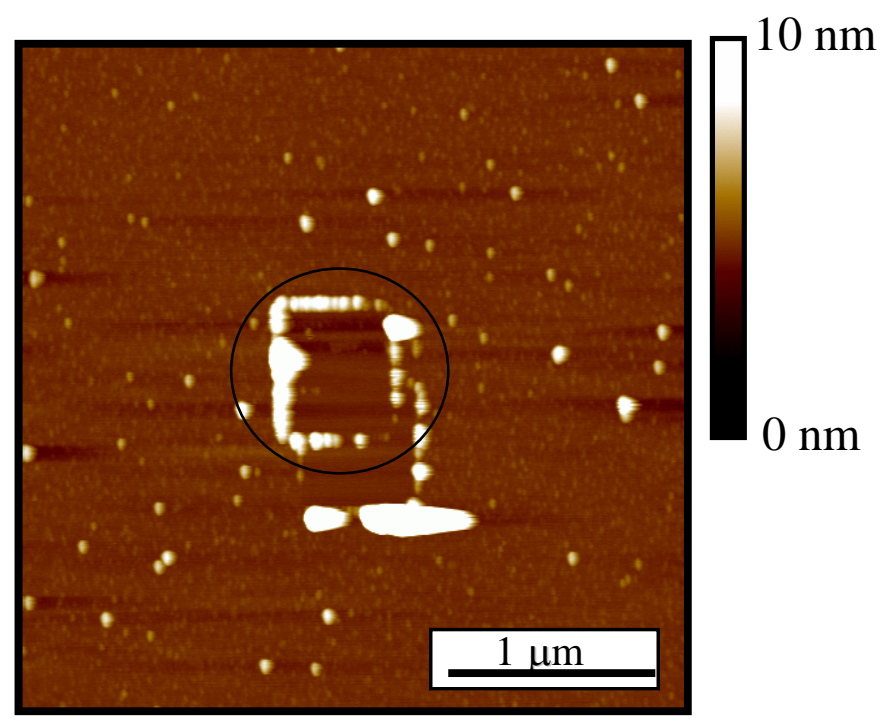

Figure S2. Tapping mode image of the same region displayed in figure 2 showing another scratched section (highlighted inside a black circle) less than $500 \mathrm{~nm}$ from the initial scratched area. This demonstrates the ease at which scratched areas can be relocated. 